Check for updates

Cite this: React. Chem. Eng., 2018, 3, 905

Received 4th September 2018, Accepted 8th October 2018

DOI: $10.1039 / c 8 r e 00194 d$

rsc.li/reaction-engineering

\title{
Intensification of esterification through emulsification: isolation of dilute low molecular weight carboxylic acids
}

\author{
Andreas Toth, iD * Susanne Lux, iD Daniela Painer and Matthäus Siebenhofer
}

\begin{abstract}
A concept for isolation of low molecular weight carboxylic acids (e.g., acetic acid) from dilute aqueous streams was developed. This concept of combining chemical conversion of the carboxylic acids with in situ liquid-liquid extraction enhanced by catalysis and emulsification was proven applicable for carboxylic acid concentration of $1 \mathrm{~mol} \mathrm{l}^{-1}$. Chemical conversion was achieved by esterification with 1-octanol, catalysed by the surfactant 4-dodecylbenzenesulfonic acid. Emulsification induced by the catalyst was confirmed to be essential for high conversion and separation efficiency. Investigations were supported and evaluated by design of experiments and yielded conversions beyond $54.3 \%$ and separation efficiencies beyond $57.5 \%$ for acetic acid. Evaluation of process parameters yielded a quadratic model for prediction of process performance. Applicability of the concept for formic acid, propionic acid and butyric acid isolation from aqueous feed was confirmed.
\end{abstract}

\section{Introduction}

Sustainable utilization of biobased feedstock to its full extent embodies one of the main aims of the biorefinery concept. This aim requires efficient and environmentally sound separation technologies. ${ }^{1}$ Such processes are widely available for feed streams with highly concentrated constituents, in sharp contrast to the isolation of constituents from dilute aqueous streams/effluents. Dilute effluent streams occur throughout the biorefinery, exemplarily in pulping processes. These processes generate effluents containing valuable constituents such as (hydroxy-)carboxylic acids at varying concentrations, which are either flushed or incinerated. ${ }^{2,3}$

A compilation of separation technologies for isolation of carboxylic acids from dilute aqueous streams is given by Talnikar et al., ${ }^{4}$ including reactive separations. The latter have shown great potential for dealing with dilute aqueous streams e.g., reactive distillation for isolation of acetic acid or formic acid. ${ }^{2,5,6}$ While these processes work well for a concentration of $30 \mathrm{wt} \%$ carboxylic acid in the feed, for lower concentrations ( $6 \mathrm{wt} \%$ and below) the only industrial applied technology is reactive extraction. ${ }^{7}$ This technology is based on adduct formation of the carboxylic acid with a reactive extractant and requires complex solvent regeneration. Sustainability and application range of such processes are criti-

Graz University of Technology, Institute of Chemical Engineering and Environmental Technology, NAWI Graz Central Lab Biobased Products, Inffeldgasse 25c/III, 8010 Graz, Austria. E-mail: andreas.toth@tugraz.at; Tel: +43 (0)316 8737460 cally lowered due to the preferred use of organophosphorous compounds and various aliphatic amines as reactive extractants. $^{4,7}$ Some of the reactive extraction processes reviewed by Talnikar et al. ${ }^{4}$ require residence times of up to $24 \mathrm{~h}$, hindering an industrial implementation.

In order to provide a simple, yet efficient alternative technology for isolation of low molecular weight carboxylic acids, the present work presents the concept of combining the two main benefits of reactive distillation and reactive extraction. These are the (catalysed) chemical conversion of reactive distillation and the moderate, easy-to-implement process of liquid-liquid extraction. In this context, chemical conversion does not refer to adduct formation in the sense of conventional reactive extraction, but rather to the transformation of the constituent to a product (e.g., esterification of carboxylic acids). For esterification, low concentration of reactants and a high excess of water inhibit the reaction rate and shift the reaction equilibrium composition to the reactant side. Nevertheless, high conversion rates may be achieved by utilizing surfactant catalysts like 4-dodecylbenzenesulfonic acid (4DBSA). The catalyst activity and its influence on the reaction was stated by Manabe et al., ${ }^{8}$ who achieved high yields in dehydrative esterification of lauric acid with 3-phenyl-1propanol in water. Emulsification induced by surfactant catalysts like 4DBSA has been reported to be beneficial for etherification reactions in water ${ }^{9}$ and various organic reactions in aqueous and biphasic media. ${ }^{8,10-12}$ Although the benefits of emulsification are highlighted e.g., for dehydration reactions, ${ }^{8}$ the work of Hohl et $a l^{12}$ indicates the complexity of emulsified multiphase systems. Thorough evaluation of 
process parameter influence on emulsification and process performance is essential for applicability and modeling of the concept.

The proposed concept of combining extraction with chemical conversion targets the limitation of the state of the art separation processes by using a reactive, catalytically active solvent phase. For this purpose, the solvent phase (e.g., $n$-undecane) contains a higher aliphatic alcohol for esterification (e.g., 1-octanol) and a surfactant catalyst (e.g., 4-dodecylbenzenesulfonic acid) for enhancing reaction rate and mass transfer. While esterification of higher molecular weight carboxylic acids is investigated more frequently, less effort has so far been spent on low molecular weight carboxylic acids, which occur more frequently in biobased effluents. The concept was thus applied for acetic acid isolation ( $1 \mathrm{~mol} \mathrm{~L}^{-1}$ ) and extended to other low molecular weight carboxylic acids. In order to meet requirements of efficient and environmentally sound separation processes, the proposed concept aims at meeting the " 24 principles of green engineering and green chemistry" summarised by Tang et al. ${ }^{13}$ and the CHEM21 selection guide for solvents by Prat et al. ${ }^{14}$

\section{Materials and methods}

\subsection{Chemicals}

Acetic acid (CAS: 64-19-7; Sigma-Aldrich) with a purity of 99100\% and 1-octanol (CAS: 111-87-5; Sigma-Aldrich) with a purity of $>98 \%$ were used as reactants. $n$-Undecane (CAS: $1120-$ 21-4; Sigma-Aldrich) with a purity of $>99 \%$ was used as diluent and 4-dodecylbenzenesulfonic acid (4DBSA; CAS: 121-65-3; Sigma-Aldrich; mixture of isomers) with a purity of $>95 \%$ was used as catalyst. Formic acid (CAS: 64-18-6; Chem-Lab), propionic acid (CAS: 79-09-4; Sigma-Aldrich) and butyric acid (CAS: 109-92-6; Merck) all with a purity of $>99 \%$ were used as reactants for reference experiments. Hydrochloric acid (CAS: 7647-01-0; Carl Roth) with a purity of $37 \%$ p.a. and Amberlite ${ }^{\circledR}$ IR120 $^{+}$(CAS: 39389-20-3; Sigma-Aldrich) were used as reference catalysts. Amberlite ${ }^{\circledR} \mathrm{IR}_{120^{+}}$was rinsed with deionized water and dried before use; all other chemicals were used without further pretreatment.

\subsection{Experimental procedure}

Esterification experiments were performed in a $1000 \mathrm{ml}$ jacket batch reactor stirred with a triple Rushton turbine impeller. For sealing the batch reactor, a custom-made PTFE-lid with sockets for the stirrer, a thermometer and for sampling was used. The batch reactor temperature was kept constant with water using a Lauda RC20 thermostat; the temperature was measured with a digital thermometer (TFA Dostmann; $\pm 0.8^{\circ} \mathrm{C}$ ). Discharging of the reaction mass was done via a PTFE-sealed outlet at the bottom of the batch reactor.

Water was filled into the batch reactor together with the solvent phase and heated up to the desired operation temperature under constant stirring. The solvent phase consisted of 1-octanol as reactant, 4-dodecylbenzenesulfonic acid (4DBSA) as catalyst and $n$-undecane as diluent. After reaching the re- action temperature, admixing the carboxylic acid initiated the reaction. The experiments were stopped after an arbitrarily chosen reaction time of $3 \mathrm{~h}$. At this time, the stirrer was stopped and after primary phase separation of $3 \mathrm{~min}$ samples of $10 \mathrm{ml}$ were taken from the top and the bottom of the reaction mixture with a syringe. These samples were centrifuged for $10 \mathrm{~min}$ to generate enough clear sample for GC analysis to avoid distorting process performance by uncontrolled reaction progress. For the mass balance, the remaining reaction mass was split by centrifugation for 30 min to achieve adequate phase separation with minimum entrainment of the two phases into each other. A Heraeus Labofuge 400 benchtop centrifuge was used for emulsion splitting at ref 2383.

\subsection{Analytics}

Both liquid phases were analysed by gas chromatography in a Shimadzu GC 2010 plus equipped with an AOC 20i/s autosampler, a flame ionization detector (FID) and a thermal conductivity detector (TCD) in parallel operation. A Phenomenex column of type Zebron ZB WAXplus $(60 \mathrm{~m} \times 0.32 \mathrm{~mm} \times$ $0.5 \mu \mathrm{m})$ was used. The samples were injected undiluted $(0.3$ $\mu \mathrm{l})$ in split mode with a split ratio of 120 and an injection temperature of $250{ }^{\circ} \mathrm{C}$. Helium was used as carrier gas. The temperature program started at $45^{\circ} \mathrm{C}$ for $5 \mathrm{~min}$ and ramped up to $150{ }^{\circ} \mathrm{C}$ with a rate of $25{ }^{\circ} \mathrm{C} \mathrm{min}^{-1}$ and a final hold for $3.3 \mathrm{~min}$. Both detectors were operated at $270{ }^{\circ} \mathrm{C}$. The relative standard error of the GC measurements was in the range of $1.9 \%$ for all components analysed via FID and $4.1 \%$ for water.

Reaction progress of the reference experiments with formic acid, propionic acid and butyric acid as well as 4DBSA and hydrochloric acid was determined with a SI Analytics TitroLine 7800 autotitrator. A potassium hydroxide solution $\left(0.1 \mathrm{~mol} \mathrm{~L}^{-1}\right)$ was used for acid/base titration. The solvent phase was dissolved in a mixture of 2-propanol and deionized water prior to titration.

\subsection{Design of experiments (DoE)}

Investigations were planned based on the concept of design of experiments in order to minimize experimental effort, while gaining insight into parameter interaction and influence. The design of experiments was developed and evaluated with the commercial software MODDE® Pro 12 (Umetrics). Table 1 shows the chosen process parameters with the investigated ranges. Carboxylic acids were used at a feed

Table 1 Investigated process parameters (factors) with minimum and maximum values

\begin{tabular}{llll}
\hline Process parameter & Min & Max & Unit \\
\hline Temperature $(T)$ & 25 & 60 & ${ }^{\circ} \mathrm{C}$ \\
Stoichiometric ratio $(\mathrm{OH}: \mathrm{H})$ & 0.8 & 1.5 & $\mathrm{~mol}_{\mathrm{OcOH}} \mathrm{mol}_{\mathrm{A}, 0}{ }^{-1}$ \\
Phase ratio $(\mathrm{S}: \mathrm{A})$ & 0.5 & 2.0 & - \\
Catalyst load $(\mathrm{CAT})$ & 0.015 & 0.060 & eq. $\mathrm{mol}_{\mathrm{A}, 0}{ }^{-1}$ \\
Stirrer speed $(n)$ & 300 & 450 & $\mathrm{rpm}$
\end{tabular}


concentration of $1 \mathrm{~mol} \mathrm{l}^{-1}$ for all experiments; the total reaction volume was fixed with $600 \mathrm{ml}$. Catalyst load and stoichiometric ratio were based on the initial amount of carboxylic acid at the reaction start.

Two characteristic numbers were defined as response factors to enable comparison and evaluation of the single experiments. The first is the overall conversion of carboxylic acid as defined in eqn (1), which allows assessment of the reaction kinetics. In this equation, $X_{\mathrm{A}}$ is the overall conversion, $m_{\mathrm{A}, 0}$ the initial mass of carboxylic acid and $m_{\mathrm{A}, \mathrm{sol}}$ and $m_{\mathrm{A}, \mathrm{aqu}}$ the mass of carboxylic acid at the end of the experiment in the solvent and in the aqueous phase, respectively.

$$
X_{\mathrm{A}}=\frac{m_{\mathrm{A}, 0}-\left(m_{\mathrm{A}, \mathrm{sol}}+m_{\mathrm{A}, \mathrm{aqu}}\right)}{m_{\mathrm{A}, 0}}
$$

The second characteristic number is the separation efficiency $\mathrm{SE}_{\mathrm{A}}$ based on the carboxylic acid (defined in eqn (2)) providing information on total carboxylic acid removal from the aqueous phase. Eqn (2) represents the separation efficiency $\mathrm{SE}_{\mathrm{A}}$, which is defined as the ratio of the mass of the carboxylic acid removed from the aqueous phase to the initial mass of carboxylic acid.

$$
\mathrm{SE}_{\mathrm{A}}=\frac{m_{\mathrm{A}, 0}-m_{\mathrm{A}, \mathrm{aqu}}}{m_{\mathrm{A}, 0}}
$$

\section{Results and discussion}

\subsection{Catalyst comparison and impact of emulsification}

Comparing different types of catalysts confirmed the necessity of emulsification for high process performance. Emulsification induced by the surfactant catalyst 4-dodecylbenzenesulfonic acid requires attention, as catalyst loads above 0.06 eq. $\operatorname{mol}_{\mathrm{A}, 0}{ }^{-1}$ produce stable emulsions. This high emulsion stability is to be avoided, since splitting of such emulsions by centrifugation is not possible. Impact of emulsification on both response factors was determined by comparing the uncatalysed esterification to esterification enhanced with three different catalysts. Hydrochloric acid and Amberlite ${ }^{\circledR}$ IR $120^{+}$were used as non-surfactant catalysts at the same molar equivalents as the surface-active catalyst 4DBSA. Attrition of the solid catalyst was not observed during the experiments due to the reactor geometry and the small amount of catalyst. Fig. 1 displays the results of these experiments with conversion in white and separation efficiency in grey.

The uncatalysed reference experiment yielded a conversion of about $4.6 \pm 0.2 \%$ and a separation efficiency of $15.9 \pm$ $0.2 \%$. These results were confirmed to be the maxima for the given process conditions as a consequence of the phase and reaction equilibria. As shown in Fig. 1, catalysis with hydrochloric acid and Amberlite ${ }^{\circledR}$ IR120 ${ }^{+}$yielded $4 \pm 0.7 \%$ and 7.7 $\pm 0.8 \%$ conversion and $15.6 \pm 0.8 \%$ and $18.9 \pm 1.6 \%$ separation efficiency, respectively. For these two catalysts, the slight

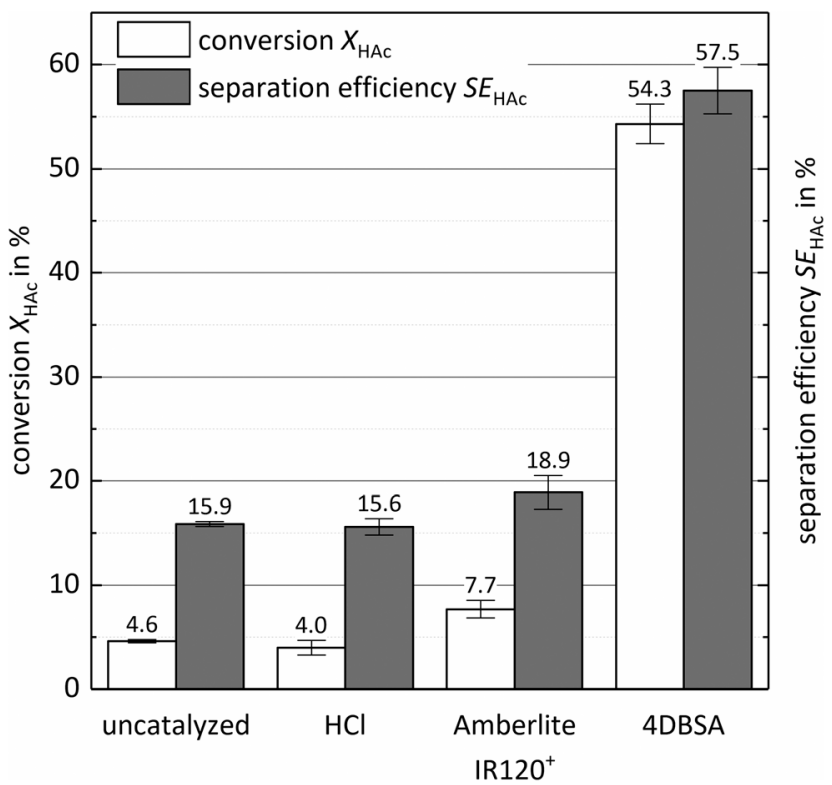

Fig. 1 Impact of emulsification induced by 4DBSA on acetic acid conversion $X_{\mathrm{HAC}}$ (left bars; white) and separation efficiency $\mathrm{SE}_{\mathrm{HAc}}$ (right bars; grey) compared to catalysis with $\mathrm{HCl}$, Amberlite ${ }^{\circledR} \mathrm{IR} 120^{+}$and an uncatalysed reference. Catalysts used at 0.06 eq. $\mathrm{mol}_{\mathrm{A}, 0^{-1}}$, reaction time $3 \mathrm{~h}$, reaction temperature $60{ }^{\circ} \mathrm{C}$, phase ratio 2 , stirrer speed 300 rpm and 1.5-fold stoichiometric excess of alcohol.

increase of $\mathrm{SE}_{\mathrm{A}}$ can be explained by the change of extraction properties of the solvent phase due to reaction progress. This improved reaction progress increases the concentration of ester in the solvent phase. The slightly better performance of the HCl-catalysed experiment compared to the uncatalysed reference is negligible and explained by a higher deviation of the $\mathrm{HCl}$-catalysed results. As a result, the catalysis with a homogeneous catalyst in the aqueous phase has no significant influence on process performance.

Comparing these results with the performance of 4DBSA confirms the positive impact of emulsification on the process. The induced emulsification highly increases the mass transfer area up to quasi-homogeneous state and thus enhances phase contact, overriding mass transfer limitation. As

Table 2 Scaled and centered parameters for the DoE-based models predicting conversion $X_{\mathrm{HAc}}$ and separation efficiency $\mathrm{SE}_{\mathrm{HAc}}$ of acetic acid esterification with a confidence interval of $95 \%$

\begin{tabular}{lrc}
\hline Parameter & \multicolumn{1}{c}{$X_{\mathrm{HAc}}$} & \multicolumn{1}{c}{$\mathrm{SE}_{\mathrm{HAc}}$} \\
\hline$\beta_{\mathrm{i}}$ & $29.13 \pm 0.72$ & $34.62 \pm 0.82$ \\
$\beta_{T}$ & $11.50 \pm 0.85$ & $10.30 \pm 0.96$ \\
$\beta_{\mathrm{S}: \mathrm{A}}$ & $0.50 \pm 0.82$ & $0.88 \pm 0.94$ \\
$\beta_{\mathrm{CAT}}$ & $4.04 \pm 0.81$ & $3.47 \pm 0.92$ \\
$\beta_{\mathrm{OH}: \mathrm{H}}$ & $3.50 \pm 0.88$ & $5.06 \pm 1.00$ \\
$\beta_{n}$ & $-1.40 \pm 0.82$ & $-1.43 \pm 0.93$ \\
$\beta_{T \cdot \mathrm{OH}: \mathrm{H}}$ & $3.80 \pm 0.97$ & $4.00 \pm 1.08$ \\
$\beta_{T \cdot n}$ & $-1.18 \pm 0.92$ & $-1.32 \pm 1.04$ \\
$\beta_{\mathrm{S}: \mathrm{A} \cdot \mathrm{CAT}}$ & $0.91 \pm 0.85$ & $1.07 \pm 0.97$ \\
$\beta_{\mathrm{S}: \mathrm{A} \cdot n}$ & $1.49 \pm 0.87$ & $1.30 \pm 0.99$ \\
$\beta_{\mathrm{CAT} \cdot \mathrm{OH}: \mathrm{H}}$ & $1.52 \pm 0.92$ & Not used \\
$\beta_{\mathrm{OH}: \mathrm{H} \cdot n}$ & $1.00 \pm 0.94$ & Not used
\end{tabular}


a result, an overall conversion of $54.3 \pm 1.9 \%$ and a separation efficiency of $57.5 \pm 2.2 \%$ was achieved with 4DBSA. As low molecular weight carboxylic acids like acetic acid are preferably present in the aqueous phase and the higher alcohol 1-octanol has a much higher solubility in the solvent phase, catalysis at the interphase or in the solvent phase is more efficient. Targeting this necessity much better than $\mathrm{HCl}$ and Amberlite ${ }^{\circledR}$ IR $120^{+}$(both mainly present in the aqueous phase), the surface active catalyst 4DBSA is assumed to accumulate at the interphase as well as in the solvent phase due to its hydrophobic dodecyl group. ${ }^{15}$

\subsection{Parameter screening and process modeling for acetic acid esterification}

Parameter screening and process modeling was done for acetic acid as model carboxylic acid. The concept of design of experiments was used to determine the main influence parameters via parameter screening and optimisation. The models shown in eqn (3) for overall conversion and in eqn (4) for separation efficiency were derived from DoE-results. Quadratic models were simplified by dismissing insignificant parameters. The remaining parameters including confidence intervals for $95 \%$ probability are given in Table 2 for both models.

$$
\begin{aligned}
X_{\mathrm{HAc}}= & \beta_{X_{\mathrm{HAc}}}+\beta_{T} \cdot(T)+\beta_{\mathrm{S}: \mathrm{A}} \cdot(\mathrm{S}: \mathrm{A})+\beta_{\mathrm{CAT}} \cdot(\mathrm{CAT}) \\
& +\beta_{\mathrm{OH}: \mathrm{H}} \cdot(\mathrm{OH}: \mathrm{H})+\beta_{n} \cdot(n)+\beta_{T \cdot \mathrm{OH}: \mathrm{H}} \cdot(T) \cdot(\mathrm{OH}: \mathrm{H}) \\
& +\beta_{T \cdot n} \cdot(T) \cdot(n)+\beta_{\mathrm{S}: \mathrm{A} \cdot \mathrm{CAT}} \cdot(\mathrm{S}: \mathrm{A}) \cdot(\mathrm{CAT})+\beta_{\mathrm{S}: \mathrm{A} \cdot n} \cdot(\mathrm{S}: \mathrm{A}) \cdot(n) \\
& +\beta_{\mathrm{CAT} \cdot \mathrm{OH}: \mathrm{H}} \cdot(\mathrm{CAT}) \cdot(\mathrm{OH}: \mathrm{H})+\beta_{\mathrm{OH}: \mathrm{H} \cdot n} \cdot(\mathrm{OH}: \mathrm{H}) \cdot(n)
\end{aligned}
$$

Twelve parameters enable prediction of acetic acid conversion with $R^{2}=0.984, Q^{2}=0.956$ and a reproducibility of 0.993. While the high $R^{2}$ indicates a good fit of the model for the experimental data, $Q^{2}$ close to 1 confirms a good response prediction within the investigated parameter ranges. Experimental noise level is very low, which is confirmed by the high reproducibility. A graphical comparison of experimental data and predicted conversions and separation efficiencies is shown in Fig. 2 and 3.

Fig. 4 (left diagram) shows the influence of scaled and centered parameters on the conversion of acetic acid. Temperature $(T)$ is the main influence parameter followed by catalyst load (CAT) and stoichiometric ratio $(\mathrm{OH}: \mathrm{H})$. Although the impact of the phase ratio ( $\mathrm{S}: \mathrm{A})$ is not significant in itself, its interaction with catalyst load and stirrer speed $(n)$ influences the overall conversion. The only parameters causing a retarding effect on the overall conversion are an increased stirrer speed and the interaction of stirrer speed and temperature. This may be explained by a shift of the drop size distribution towards smaller droplet diameters. The surface of small droplets becomes rigid due to the surfactant molecules and thus hinders mass transfer. ${ }^{15}$

$$
\begin{aligned}
\mathrm{SE}_{\mathrm{HAc}}= & \beta_{\mathrm{SE}_{\mathrm{HAc}}}+\beta_{T} \cdot(T)+\beta_{\mathrm{S}: \mathrm{A}} \cdot(\mathrm{S}: \mathrm{A})+\beta_{\mathrm{CAT}} \cdot(\mathrm{CAT}) \\
& +\beta_{\mathrm{OH}: \mathrm{H}} \cdot(\mathrm{OH}: \mathrm{H})+\beta_{n} \cdot(n)+\beta_{T \cdot \mathrm{OH}: \mathrm{H}} \cdot(T) \cdot(\mathrm{OH}: \mathrm{H}) \\
& +\beta_{T \cdot n} \cdot(T) \cdot(n)+\beta_{\mathrm{S} \mathrm{AA} \cdot \mathrm{CAT}} \cdot(\mathrm{S}: \mathrm{A}) \cdot(\mathrm{CAT})+\beta_{\mathrm{S}: \mathrm{A} \cdot n} \cdot(\mathrm{S}: \mathrm{A}) \cdot(n)
\end{aligned}
$$

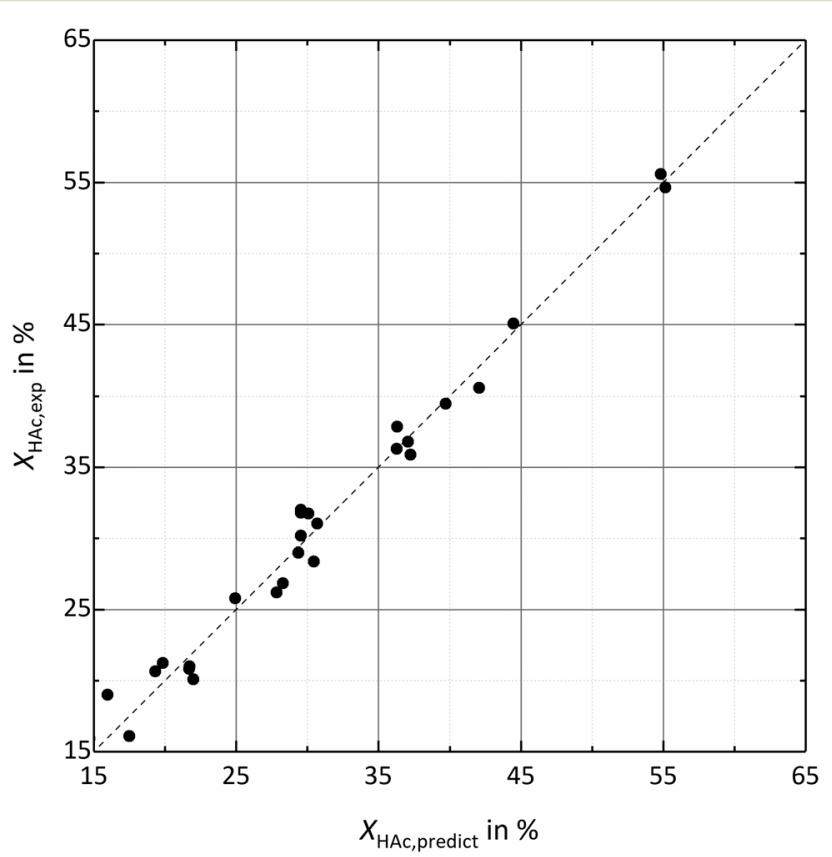

Fig. 2 Comparison of experimental conversion $X_{\text {HAc,exp }}$ and predicted conversion $X_{\text {HAc,predict }}$ based on the DoE-model $\left(R^{2}=0.984 ; Q^{2}=\right.$ 0.956; reproducibility $=0.993$ )

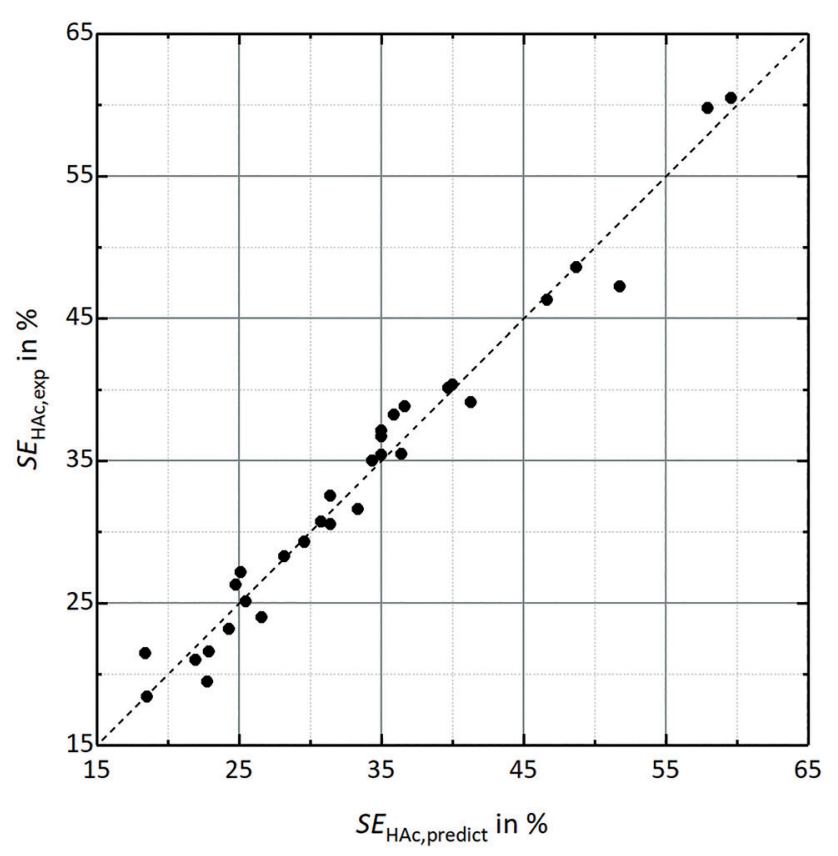

Fig. 3 Comparison of experimental separation efficiency $\mathrm{SE}_{\mathrm{HAc}, \exp }$ and predicted separation efficiency $\mathrm{SE}_{\mathrm{HAc} \text {,predict }}$ based on the DoE-model $\left(R^{2}=0.974 ; Q^{2}=0.931 ;\right.$ reproducibility $\left.=0.993\right)$. 

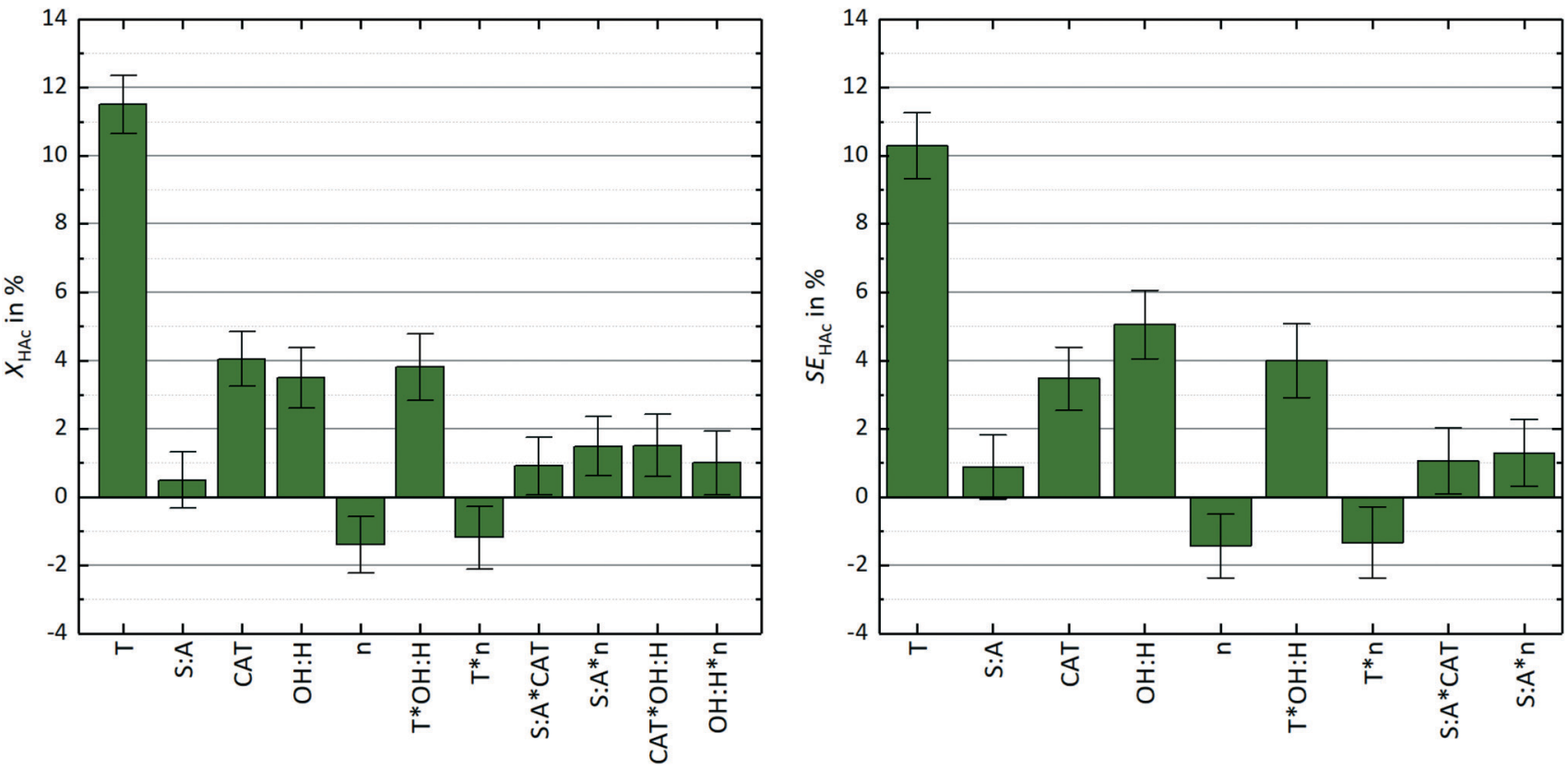

Fig. 4 Coefficient plot for the DoE-models showing their influence on conversion $X_{\mathrm{HAc}}$ (left-hand side plot) and separation efficiency $\mathrm{SE} \mathrm{HAc}_{\mathrm{HC}}$ (righthand side plot) of acetic acid esterification with a confidence interval of $95 \%$.

Similar results were found for the separation efficiency, which is influenced by 9 parameters as shown in eqn 4 and Table 2. In contrast to the overall conversion, the interaction between catalyst load and stoichiometric ratio $\left(\beta_{\mathrm{CAT} \cdot \mathrm{OH}: \mathrm{H}}\right)$ and stoichiometric ratio and stirrer speed $\left(\beta_{\mathrm{OH}: \mathrm{H} \cdot n}\right)$ has no significant impact on $\mathrm{SE}_{\mathrm{A}}$. Nevertheless, the model predicts the separation efficiency as displayed in the diagram in Fig. 3 very well. This is confirmed by a $R^{2}$ of 0.974 , a $Q^{2}$ of 0.931 and a reproducibility of 0.993 .

The right-hand side diagram in Fig. 4 gives an overview of the parameter influence on the separation efficiency including the $95 \%$-confidence intervals. Reaction temperature is still the most important parameter, but compared to the overall conversion it has slightly less impact on separation efficiency. By contrast, the influence of the stoichiometric ratio is increased for the separation efficiency, as it corresponds with the composition and therefore the extraction capacity of the solvent phase. The limiting effect of an increased stirrer speed is observed in the same magnitude as for overall conversion.

3.2.1 Interpretation of parameter influence on acetic acid separation efficiency via contour plot analysis. Fig. 5 illustrates the predicted separation efficiency in dependence on all investigated process parameters in a contour plot. Within this contour plot, three effects are revealed. The first effect is a trend of rising separation efficiency from the lower left corner (all parameters at minimum level; plot A3) to the upper right corner (all parameters at maximum level; plot C1). High $\mathrm{O}: \mathrm{H}, \mathrm{CAT}$ and $T$ are expected to increase conversion and thereby positively influence the extraction towards higher separation efficiencies and thus confirming the observed trend.
A second effect is the enhanced mass transfer due to a high catalyst load which is best observed in plot C1 of Fig. 5 . With a $\mathrm{CAT}_{\max }$ of 0.06 eq. $\operatorname{mol}_{\mathrm{A}, 0}{ }^{-1}$ even at a stoichiometric ratio of 0.8 and nearly ambient temperature separation efficiencies in the range of $35 \%$ are possible at a stirrer speed of $475 \mathrm{rpm}$. The latter leads to the third effect: decreased separation efficiency for high stirrer speeds. This effect is assumed to be caused by too small droplets with highly rigid surfaces due to the surfactant (catalyst) molecules. Such rigid surfaces increasingly hinder mass transfer, hence limiting the separation efficiency and boosting unwanted emulsion stability.

3.2.2 Mass transfer capacity limitation due to exceeding extraction capacity of the solvent phase. Two experiments ( $T$ $=60{ }^{\circ} \mathrm{C}, \mathrm{CAT}=0.06$ eq. $\mathrm{mol}_{\mathrm{A}, 0}{ }^{-1}, \mathrm{OH}: \mathrm{H}=1.5, n=300 \mathrm{rpm}$ ) differed only in phase ratio with one at minimum $\mathrm{S}: \mathrm{A}_{\min }=$ 0.5 and the other at maximum S : $\mathrm{A}_{\max }=2$. Evaluation after 3 $\mathrm{h}$ reaction time revealed nearly the same results for both experiments $\left(X_{\mathrm{HAc}} \approx 55 \% ; \mathrm{SE}_{\mathrm{HAc}} \approx 60 \%\right)$. The thereby indicated mass transfer limitation could be confirmed by extending reaction time for both experiments to $12 \mathrm{~h}$. While both response factors remained nearly unchanged for $\mathrm{S}: \mathrm{A}_{\min }\left(X_{\mathrm{HAc}}\right.$ $\approx 57 \% ; \mathrm{SE}_{\mathrm{HAc}} \approx 63 \%$ ), a significant increase was achieved for $\mathrm{S}: \mathrm{A}_{\max }$ to $X_{\mathrm{HAc}} \approx 74 \%$ and $\mathrm{SE}_{\mathrm{HAc}} \approx 75 \%$. This increase supports the necessity of higher phase ratios in order to avoid exceeding the solvent's extraction capacity. In addition, the difference between conversion and separation efficiency for the maximum phase ratio experiment decreases. This implies approaching both reaction and extraction equilibrium in the system.

3.2.3 Increase of the reaction temperature to $75^{\circ} \mathrm{C}$ and 95 ${ }^{\circ} \mathrm{C}$. A further approach to increase both response factors was 

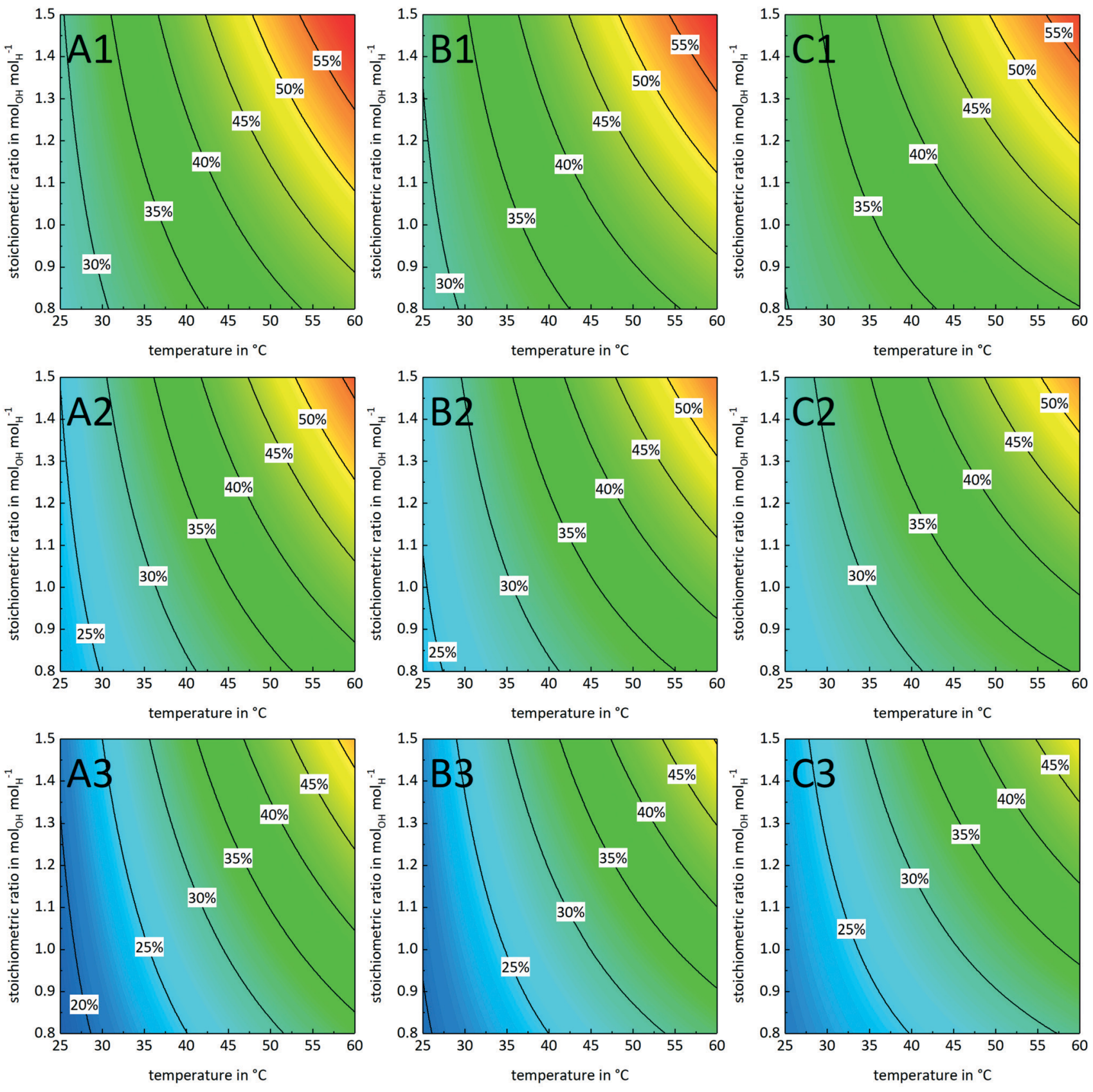

$15 \%$

$20 \%$

$25 \%$

$30 \%$

$35 \%$

$40 \%$

$45 \%$

$50 \%$

$55 \%$

$60 \%$

Fig. 5 Contour plot of predicted acetic acid separation efficiency $\mathrm{SE}_{\mathrm{HAc} \text {, predict }}$ in\% for a fixed phase ratio $\mathrm{S}: \mathrm{A}$ of 2 in dependency of stirrer speed (columns A, B and C), catalyst load (row 1, 2 and 3), stoichiometric ratio and temperature. Column A: 300 rpm, column B: 375 rpm, column C: 450 rpm; row 1: 0.06 eq. mol $_{A, 0}{ }^{-1}$, row 2: 0.03 eq. mol $_{A, 0}{ }^{-1}$, row 3: 0.015 eq. mol $_{A, 0}{ }^{-1}$.

increasing the reaction temperature from $60{ }^{\circ} \mathrm{C}$ to $75^{\circ} \mathrm{C}$ and $95^{\circ} \mathrm{C}$, exceeding the limits of the DoE-set. All process parameters were kept constant at CAT $=0.06$ eq. $\operatorname{mol}_{\mathrm{A}, 0}{ }^{-1}, \mathrm{OH}: \mathrm{H}=$ $1.5, n=300 \mathrm{rpm}$ and $\mathrm{S}: \mathrm{A}=2$ for $3 \mathrm{~h}$ reaction time. A reaction temperature of $75{ }^{\circ} \mathrm{C}$ yielded an overall conversion of $70.8 \pm$ $0.0 \%$ and a separation efficiency of $72.8 \pm 0.1 \%$. Compared to this, $95{ }^{\circ} \mathrm{C}$ provided $73.7 \pm 0.7 \%$ conversion and $75.5 \pm 1.1 \%$ separation efficiency. From these results, two facts can be observed: on the one hand, a temperature increase above $75^{\circ} \mathrm{C}$ does not yield a significant increase in either response factor. On the other hand, a temperature of $95{ }^{\circ} \mathrm{C}$ offers the possibility of decreasing the reaction time, as the system approaches its equilibrium conversion faster.

Additionally enhancing the extraction would shift the equilibrium composition to the product side and eliminate mass transfer limitation. Thus, a multi-step process similar to a mixer-settler cascade in counter-current operation is proposed. The multi-stage process concept maximises extraction 
Table 3 Partition coefficients and corresponding separation efficiencies for investigated low molecular weight carboxylic acids

\begin{tabular}{lll}
\hline Carboxylic acid & $\log K_{\mathrm{OW}}{ }^{16}$ & $\mathrm{SE}_{\mathrm{i}}$ \\
\hline Formic acid & -0.54 & $61.4 \pm 2.3 \%$ \\
Acetic acid & -0.17 & $57.5 \pm 2.2 \%$ \\
Propionic acid & 0.33 & $70.5 \pm 2.7 \%$ \\
Butyric acid & 0.79 & $79.6 \pm 0.4 \%$
\end{tabular}

driving force and may give access to nearly complete conversion. Finding an optimal temperature range with respect to conversion and reaction time requires further investigation.

\subsection{Esterification of other low molecular weight carboxylic acids}

The 4DBSA-catalysed concept was applied to other low molecular weight carboxylic acids, namely formic acid, propionic acid and butyric acid. Experiments were performed at $60{ }^{\circ} \mathrm{C}$, CAT $=0.06$ eq. $\operatorname{mol}_{\mathrm{A}, 0}{ }^{-1}, \mathrm{OH}: \mathrm{H}=1.5, n=300 \mathrm{rpm}$ and $\mathrm{S}: \mathrm{A}=$ 2 for $3 \mathrm{~h}$. The concept was proved to be applicable for the investigated carboxylic acids, which is best displayed by the separation efficiencies. Separation efficiencies were $61.4 \pm$ $2.3 \%$ for formic acid, $70.5 \pm 2.7 \%$ for propionic acid and 79.6 $\pm 0.4 \%$ for butyric acid compared to $57.5 \pm 2.2 \%$ for acetic acid. Increasing chain length of the carboxylic acid and thus decreasing $\log K_{\mathrm{OW}}$-values result in increasing separation efficiency, except for formic acid as displayed in Table 3. Although formic acid prefers the aqueous phase based on its $\log K_{\mathrm{OW}}$-value, a higher reactivity compared to acetic acid allows a higher separation efficiency.

\section{Conclusions}

In accordance with the " 24 principles of green engineering and green chemistry", ${ }^{13}$ a concept was proposed to prevent loss of unused carboxylic acids from renewable resources. This is achieved by using catalytic reagents for isolation at moderate temperatures and ambient pressure with low toxicity of the products in a safe and simple process. The concept of utilizing emulsification with the surfactant-catalyst 4-dodecylbenzenesulfonic acid in order to enhance the reaction rate for isolation of low molecular weight carboxylic acids via esterification with 1-octanol was confirmed in single-stage batch operation. Further improvement of process performance can be achieved by multi-stage operation with adequate phase separation between the reaction stages.

\section{Indices and symbols}

\section{Indices}

$\begin{array}{ll}\text { A } & \text { Acid } \\ 0 & \text { Initial } \\ \text { sol } & \text { Solvent phase } \\ \text { aqu } & \text { Aqueous phase } \\ \text { exp } & \text { Experimental } \\ \text { predict } & \text { Predicted }\end{array}$

predict Predicted
HAc Acetic acid

$\mathrm{OcOH}$ 1-Octanol

\section{Symbols}

$X \quad$ Conversion

SE $\quad$ Separation efficiency

$\beta \quad$ Parameter of the models derived by design of experiments

DoE Design of experiments

4DBSA 4-Dodecylbenzenesulfonic acid

HCl Hydrochloric acid

$R^{2} \quad$ Coefficient of determination

$Q^{2} \quad$ Percent of variation

$\log K_{\text {OW }} n$-Octanol/water partition coefficient

\section{Conflicts of interest}

There are no conflicts to declare.

\section{Acknowledgements}

The authors gratefully acknowledge the contribution of Andrea Stachel and Michael Christopharo in performing the experimental work.

\section{References}

1 B. Kamm, P. R. Gruber and M. Kamm, BiorefineriesIndustrial Processes and Products, Wiley-VCH Verlag $\mathrm{GmbH}$, Weinheim, Germany, 2005.

2 D. Painer, S. Lux, A. Grafschafter, A. Toth and $\mathbf{M}$. Siebenhofer, Chem. Ing. Tech., 2017, 89, 161-171.

3 M. Hundt, Ph.D Thesis, Brandenburgischen Technischen Universität Cottbus-Senftenberg, 2015.

4 V. D. Talnikar and Y. S. Mahajan, Korean J. Chem. Eng., 2014, 31, 1720-1731.

5 B. Saha, S. P. Chopade and S. M. Mahajani, Catal. Today, 2000, 60, 147-157.

6 H. T. R. TEO and B. Saha, J. Catal., 2004, 228, 174-182.

7 W. Kanzler and J. Schedler, AT 365 080, 1981.

8 K. Manabe, S. Iimura, X.-M. Sun and S. Kobayashi, J. Am. Chem. Soc., 2002, 124, 11971-11978.

9 S. Mandal, S. Mandal, S. K. Ghosh, P. Sar, A. Ghosh, R. Saha and B. Saha, RSC Adv., 2016, 6, 69605-69614.

10 S. Kobayashi and K. Manabe, Acc. Chem. Res., 2002, 35, 209-217.

11 M. Shiri and M. A. Zolfigol, Tetrahedron, 2009, 65, 587-598.

12 L. Hohl, M. Knossalla and M. Kraume, Chem. Eng. Sci., 2017, 171, 76-87.

13 S. Y. Tang, R. A. Bourne, R. L. Smith and M. Poliakoff, Green Chem., 2008, 10, 268.

14 D. Prat, A. Wells, J. Hayler, H. Sneddon, C. R. McElroy, S. AbouShehada and P. J. Dunn, Green Chem., 2016, 18, 288-296.

15 T. F. Tadros, Emulsion Formation and Stability, Wiley-VCH Verlag GmbH \& Co. KGaA, Weinheim, Germany, 2013.

16 CRC handbook of chemistry and physics: A ready-reference book of chemical and physical data, ed. D. R. Lide, CRC Press, Boca Raton, 84th edn, 2003. 